\title{
Le profil social des maires de France
}

Version auteur publiée dans le $\mathrm{n}^{\circ} 148$ (janvier 2014) de la revue Pouvoirs, revue française d'études constitutionnelles et politiques (p. 123-138).

Par Michel Koebel, professeur de sociologie à l'Université de Strasbourg.

Les études fondatrices de Daniel Gaxie, inspirées des analyses de Pierre Bourdieu, sur le personnel politique avaient mis en évidence dès le début des années 1980 une sélection sociale d'autant plus forte que l'on s'élève dans la hiérarchie politique ${ }^{1}$. Depuis, bien peu d'analyses statistiques ont porté sur les élus municipaux, alors même qu'ils sont les plus nombreux, que l'on s'intéresse à l'ensemble des conseillers municipaux (plus de 500 000) ou seulement aux maires (près de 37 000). Si l'on en restait au principe énoncé par Gaxie, ces élus - puisque placés tout en bas de la hiérarchie des élus - devraient logiquement être les plus représentatifs (au sens statistique) de la population qu'ils sont censés représenter. L'objectif de cet article est de vérifier cette hypothèse à propos des élus locaux actuels - et plus particulièrement des maires - grâce aux plus récentes données concernant ces élus, en prenant en compte différents critères et en replaçant les maires dans leurs contextes : au sein de leur conseil municipal - vis à vis de leurs adjoints et des autres conseillers municipaux; selon la taille de la commune; selon leur sexe, leur âge, leur appartenance partisane, etc. Cet exercice comporte cependant certaines limites liées à la disponibilité de sources. Le Répertoire national des élus (RNE) a considérablement diversifié les données disponibles, mais ces dernières ne concernent que les élections de 2008 (caractériser les élus actuels, c'est aussi les comparer à leurs prédécesseurs). De plus, certaines difficultés méthodologiques ne sont toujours pas levées, et notamment la relative incompatibilité des catégories socioprofessionnelles utilisées pour les élus par rapport à celles de l'INSEE, sans oublier que les données du RNE reposent sur les déclarations des candidats et, le plus souvent, sur leurs propres compétences à choisir la catégorie adéquate parmi les 69 proposées, ce qui conduit immanquablement à des erreurs (volontaires ou non d'ailleurs, et entrant parfois dans des stratégies de présentation de soi inhérentes à ce que représente une candidature publique à des élections) dont personne aujourd'hui ne peut évaluer l'ampleur².

L'élaboration d'un profil social des maires de France ne doit cependant pas être perçue seulement comme une étude statistique au sens strict. Cette sélection sociale est non seulement le produit de processus électoraux et des choix français en matière modes de scrutin qui en forment le cœur, mais elle est également liée à une façon de concevoir l'accès au monde politique : aucune formation préalable possible (ou presque) en dehors d'une socialisation par les pairs - et d'abord par la famille. Ce mode d'entrée en politique produit les inégalités que l'on connaît, notamment dans le sentiment de compétence à briguer ou exercer un mandat politique. Les différences de compétences acquises via la formation initiale ou par l'expérience professionnelle ne font que renforcer par la suite ces inégalités, parce que les ressources réutilisables en politique - sur le plan symbolique (avoir fait l'ENA ou Science po) comme sur le plan pratique (maitriser la parole en public, savoir communiquer, savoir gérer des dossiers complexes et manager des équipes) - ne sont généralement acquises que dans les formations de plus haut niveau et dans des professions faisant partie du pôle supérieur de la hiérarchie sociale.

Enfin, il ne faut pas minimiser les effets d'une appartenance sociale majoritaire de ceux qui concentrent le pouvoir - fût-il local - sur le contenu même des politiques qu'ils mènent et sur leur capacité à représenter d'autres catégories de population que la leur ou que celles qui savent se faire entendre (et qui sont rarement éloignées de la leur) : une appartenance sociale produit des pensées et des représentations spécifiques, y compris en politique, et il faudrait aux élus de grandes capacités de socioanalyse et de décentration, et beaucoup de temps - ce qu'ils n'ont jamais - pour espérer tenter de prendre la distance nécessaire à une réflexion sur la notion d'intérêt général - si elle existe - et sa mise en pratique dans des politiques adaptées.

\footnotetext{
${ }^{1}$ Et notamment : Gaxie (Daniel), « Les logiques du recrutement politique », Revue française de sciences politiques, 1/30, février 1980.

${ }^{2}$ Que soient remerciés ici les fonctionnaires de la Direction générale des collectivités locales du Ministère de l'intérieur qui, depuis 10 ans, me fournissent gratuitement les données qui m'ont permis de produire ces analyses, et ce, sans que mes recherches soient estampillées d'un quelconque contrat de recherche (ce qui n'est pas sans effet sur ma liberté d'analyse).
} 


\section{Des variations socioprofessionnelles chevillées à la taille des communes}

De toutes les variables étudiées, celle qui discrimine le plus socialement les maires (c'est d'ailleurs le cas aussi de leurs adjoints et de tous les conseillers municipaux) est la taille de la commune (cf. Graphique 1). Certes les agriculteurs exploitants restent toujours la catégorie la plus surreprésentée dans les communes françaises, mais leur hégémonie (ils représentent plus de $25 \%$ des maires de France alors que leur poids dans la population est inférieur à $2 \%$ ) est inversement proportionnelle à la taille des communes, à tel point que leur présence devient négligeable voire nulle au-delà de 10000 habitants (on peut y ajouter le fait que cette catégorie est en perte de pouvoir puisqu'en 1983, elle représentait encore plus de $45 \%$ des maires). Pourtant, toutes tailles de commune confondues, ce sont les cadres et professions intellectuelles supérieures qui dominent la fonction : $28,5 \%$ des maires font partie de cette catégorie (alors que cette dernière ne représentent que $15,3 \%$ de la population). Mais leur domination est exactement inverse de celle des agriculteurs : elle est parfaitement proportionnelle à la taille de la commune, jusqu'à atteindre $93 \%$ dans les plus grandes villes. Or le prestige de la fonction (celle de maire mais également des adjoints et des « simples ${ }^{3} »$ conseillers municipaux) et les avantages matériels qui y sont liés sont proportionnels à cette taille.

Ainsi les postes les plus prestigieux sont accaparés par les cadres supérieurs, qui semblent délaisser plus facilement aux autres catégories sociales les villages, et ce, d'autant plus qu'ils sont petits. On peut même aller jusqu'à dire qu'une catégorie sociale est d'autant plus dominante qu'elle parvient à placer ses membres à la tête des communes les plus grandes.

\section{Graphique 1 : catégorie socioprofessionnelle des maires selon la taille des communes ${ }^{4}$}

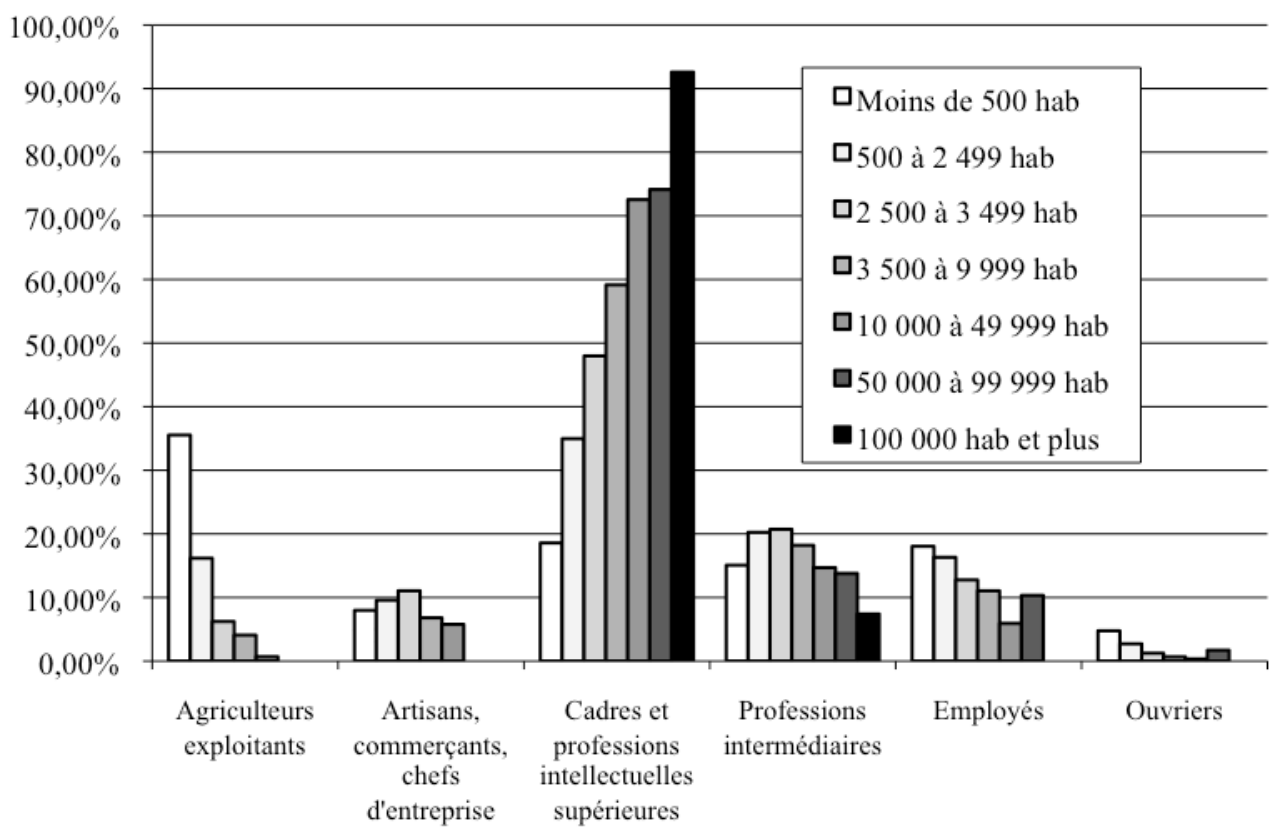

À l'autre bout de l'échelle sociale, les ouvriers sont toujours aussi mal lotis quant à leur accès aux plus hautes fonctions municipales (comme dans le reste des conseils municipaux d'ailleurs): alors qu'ils représentent encore $23,5 \%$ de la population (quand on ne prend en compte que les actifs ayant un emploi), ce n'est que dans les communes de moins de 500 habitants qu'ils parviennent à une représentation significative (4,8\% des maires) ; dans l'ensemble des communes dépassant 2500 habitants, on ne dénombre en tout et pour tout que 19 maires ouvriers pour 2474 postes à pourvoir $(0,8 \%)$, alors qu'on y trouve 1486

\footnotetext{
${ }^{3}$ Le qualificatif de « simple » conseiller municipal, parfois appelé aussi « ordinaire », n'est pas tout à fait exact puisque les tout premiers de la ou des listes vaincues - que l'on appelle souvent « l'opposition » - en font partie aussi.

${ }^{4}$ Le choix a été fait de ne retenir que les 6 premiers postes des 8 catégories de l'INSEE), seules à même de refléter la hiérarchie sociale (notamment pour les quatre dernières). Dans l'ordre et de gauche à droite: Agriculteurs exploitants; Artisans, commerçants, chefs d'entreprise ; Cadres et professions intellectuelles supérieures ; Professions intermédiaires ; Employés ; Ouvriers.
} 
maires cadres supérieurs $(60,1 \%)$. Les employés sont dans une situation différente : certes leur présence parmi les maires a régulièrement augmenté depuis 1983, en passant de 6,9 à 16,6\% entre 1983 et 2008, leur poids relatif ayant ainsi plus que doublé ; mais ils restent néanmoins largement sous-représentés par rapport à la population des actifs (29,3\%), et, à l'instar de la plupart des autres catégories - hormis l'exception notoire des cadres et professions intellectuelles supérieures - leur poids est surtout lié à leur présence dans les petites communes, là où les enjeux - et les bénéfices réels et potentiels - sont les plus faibles.

\section{Évolution et poids relatif des catégories socioprofessionnelles des maires}

Les données présentées ont volontairement écarté de l'analyse les retraités (qui représentent pourtant près du tiers des maires de France) et les « autres » catégories, qui ne recoupent d'ailleurs pas exactement la $8^{\mathrm{e}}$ catégorie de l'INSEE, et dans laquelle ont été classés les « autres professions » (1664 maires), les étudiants (14) et les «sans profession déclarée » (703). En effet, les données du RNE comportent encore d'autres zones d'ombre que celles déjà évoquées, qu'il est parfois impossible d'éclairer, surtout si l'on focalise l'analyse sur la position des élus dans une hiérarchie socioprofessionnelle. Ainsi, aucun détail n'est disponible sur la dernière profession exercée des retraités, ce qui semble rendre impossible une analyse sérieuse de cette catégorie. Il est toutefois possible d'atténuer ce qui a été souvent décrit comme une très forte poussée des retraités parmi les maires : ils ont certes doublé en nombre de 1983 à 2008 en passant de $6063(16,6 \%)$ à $11528(32,2 \%)$, mais la part des retraités dans la population a elle aussi augmenté - et dans les mêmes proportions - au cours de cette période, passant de 7,4 millions (13,6\%) en 1982 à 13,8 millions (26,2\%) en 2010.

Concernant les autres catégories socioprofessionnelles des maires, il est utile là aussi de relativiser leur évolution au cours des quatre derniers mandats (1983 à 2008), en les pondérant selon l'évolution de chaque catégorie. Cette perspective a pour conséquence d'atténuer l'augmentation numérique impressionnante des maires employés (passant de 2020 à 3639, ce qui correspond à une augmentation de 80\%) : leur proportion au sein de la catégorie n'augmente que de 67\% (cf. Tableau 1). L'effet est plus important encore sur les catégories les plus aisées, puisque les tendances vont même jusqu'à s'inverser : le poids numérique des maires cadres et professions intellectuelles supérieures est certes en forte augmentation (passant de 5493 à 6241) mais leur présence pondérée dans leur catégorie diminue (passant de 289 à 210 pour 100000 ). Inversement, les maires agriculteurs semblent perdre moins d'influence que ce que la chute de leurs effectifs aurait pu laisser penser (ils passent de 13260 à 5648 en l'espace de 25 ans, mais passent seulement de 901 à 667 pour 100000 agriculteurs, du fait de la chute vertigineuse des effectifs de cette profession au cours des 50 dernières années).

\section{Tableau 1 : Nombre de maires pour 100000 personnes de la CSP correspondante 5}

\begin{tabular}{|l|r|r|r|}
\hline Catégorie socioprofessionnelle (6 postes) & \multicolumn{1}{|c|}{$\mathbf{1 9 8 3}$} & \multicolumn{1}{c|}{$\mathbf{1 9 9 5}$} & \multicolumn{1}{c|}{$\mathbf{2 0 0 8}$} \\
\hline Agriculteurs exploitants & 901,5 & 848,7 & 667,2 \\
\hline Artisans, commerçants, chefs d'entreprise & 206,7 & 144,0 & 103,4 \\
\hline Cadres et professions intellectuelles supérieures & 289,4 & 216,8 & 210,6 \\
\hline Professions intermédiaires & 94,4 & 80,1 & 70,0 \\
\hline Employés & 32,3 & 37,8 & 48,1 \\
\hline Ouvriers & 10,2 & 9,0 & 10,7 \\
\hline
\end{tabular}

Si l'on rentre un peu plus dans le détail des catégories socioprofessionnelles, on s'aperçoit que plusieurs catégories de professions traditionnellement considérées comme pourvoyeuses de notables locaux, à l'instar des médecins et des notaires, sont en très net recul : les professions médicales de type libéral (hors salariés) baissent de plus de $40 \%$ en 25 ans ; les notaires (et avoués, huissiers, greffiers) sont en recul de $62 \%$ (seuls les avocats réussissent à se maintenir), comme les commerçants $(-65 \%)$, les artisans $(-47 \%)$, les entrepreneurs en bâtiments (-74\%), les industriels et chefs d'entreprises ( $-29 \%)$ et même les enseignants

\footnotetext{
${ }^{5}$ Les effectifs des maires proviennent du Ministère de l'Intérieur. Les données des populations de référence sont issues de l'INSEE (population active de 15 ans et plus) : RP 1982 pour les maires élus en 1983 ; moyenne des RP 1990 et 1999 pour ceux élus en 1995 et RP 2010 pour ceux élus en 2008.
} 
(-25\%). À l'inverse, la plupart des salariés sont en hausse - parfois très forte - comme les salariés du secteur médical $(+132 \%)$. Toutes les catégories de fonctionnaires sont en forte hausse (même s'il faut tenir compte là aussi de l'augmentation de cette catégorie) : fonctionnaires de catégorie A $(+91 \%), \mathrm{B}(+57 \%)$, et C (+99\%); mais également la plupart des catégories de salariés du secteur privé (cadres supérieurs $+79 \%$, autres cadres $+36 \%$ et employés $+83 \%)$, à l'exception des ouvriers de ce secteur $(-36 \%)$.

Cependant, cette tentative de relativisation des données brutes ne doit pas occulter l'hégémonie des classes supérieures sur les postes importants. En effet, les données disponibles concernant les maires sur plusieurs mandatures confondent allègrement les quelques centaines de villes moyennes et grandes et les dizaines de milliers de villages de moins de 1000 habitants, dont l'importance politique en terme de carrière est le plus souvent négligeable (sauf à être couplée à d'autres mandats plus glorieux). Ainsi, lorsque l'on observe de près les fonctionnaires, on s'aperçoit que leur présence dans les petites communes est inversement proportionnelle à leur rang (cf. Tableau 2). Dans le même ordre d'idée, la forte augmentation des employés parmi les maires a surtout concerné les petites communes (les plus nombreuses) puisque la quasi totalité d'entre eux (97\%) dirigent des communes de moins de 3500 habitants (et plutôt les plus petites d'entre elles).

Tableau 2 : Les maires fonctionnaires (2008) selon la taille de la commune (effectifs et \%)

\begin{tabular}{|c|c|c|c|c|c|c|c|c|c|}
\hline Fonctionnaires & $\begin{array}{c}\text { Moins } \\
\text { de } 100 \\
\text { hab. }\end{array}$ & $\begin{array}{l}100 \text { à } \\
499 \\
\text { hab. }\end{array}$ & $\begin{array}{l}500 \text { à } \\
1499 \\
\text { hab. }\end{array}$ & $\begin{array}{c}1500 \text { à } \\
2499 \\
\text { hab. }\end{array}$ & $\begin{array}{c}2500 \text { à } \\
3499 \\
\text { hab. }\end{array}$ & $\begin{array}{c}3500 \text { à } \\
9999 \\
\text { hab. }\end{array}$ & $\begin{array}{c}10000 \text { à } \\
19999 \\
\text { hab. }\end{array}$ & $\begin{array}{c}20000 \text { à } \\
99999 \\
\text { hab. }\end{array}$ & $\begin{array}{c}100000 \\
\text { hab. et } \\
\text { plus }\end{array}$ \\
\hline \multirow{2}{*}{$\begin{array}{l}\text { Grands corps de } \\
\text { l'Etat }\end{array}$} & 1 & 11 & 10 & 6 & 3 & 13 & 4 & 17 & 5 \\
\hline & $1,43 \%$ & $15,71 \%$ & $14,29 \%$ & $8,57 \%$ & $4,29 \%$ & $18,57 \%$ & $5,71 \%$ & $24,29 \%$ & $7,14 \%$ \\
\hline \multirow{2}{*}{ Catégorie A } & 27 & 216 & 194 & 58 & 29 & 93 & 29 & 27 & 2 \\
\hline & $4,00 \%$ & $32,00 \%$ & $28,74 \%$ & $8,59 \%$ & $4,30 \%$ & $13,78 \%$ & $4,30 \%$ & $4,00 \%$ & $0,30 \%$ \\
\hline \multirow{2}{*}{ Catégorie B } & 54 & 239 & 177 & 34 & 20 & 38 & 8 & 6 & 0 \\
\hline & $9,38 \%$ & $41,49 \%$ & $30,73 \%$ & $5,90 \%$ & $3,47 \%$ & $6,60 \%$ & $1,39 \%$ & $1,04 \%$ & $0,00 \%$ \\
\hline \multirow{2}{*}{ Catégorie $\mathrm{C}$} & 98 & 317 & 138 & 21 & 8 & 13 & 4 & 1 & 0 \\
\hline & $16,33 \%$ & $52,83 \%$ & $23,00 \%$ & $3,50 \%$ & $1,33 \%$ & $2,17 \%$ & $0,67 \%$ & $0,17 \%$ & $0,00 \%$ \\
\hline
\end{tabular}

Une autre croyance est régulièrement relayée par les médias : la « surreprésentation des fonctionnaires dans les instances politiques ${ }^{6}$ » du fait du statut plus favorable de la fonction publique au regard des élections. Pourtant, le pourcentage actuel des maires concernés $\left(18,1 \%\right.$ de maires sont issus de la fonction publique $\left.{ }^{7}\right)$ reste inférieur à celui des fonctionnaires dans la population active ayant un emploi (20,1\%). Certes, comme on l'a vu plus haut, ce ne sont pas massivement des "petits fonctionnaires » qui deviennent premiers magistrats, mais, dans le privé, ce ne sont pas non plus les plus modestes d'entre eux qui occupent généralement cette fonction.

\section{Des maires qui dominent socialement leurs conseillers municipaux}

Les données disponibles via le RNE permettent également - et pour la première fois au-delà des simples données d'âge et de sexe - de positionner socialement les maires dans leur environnement d'élus, c'est-àdire au sein de leurs conseils municipaux, en permettant notamment de les différencier des adjoints et des « simples » conseillers municipaux. Il est théoriquement possible d'observer ces données dans chacune des communes de France, mais ce qui nous intéresse ici est d'avoir une vision globale sur les différences significatives entre ces populations pour dégager des tendances (et qui ne seront évidemment pas vérifiées dans l'ensemble des communes de France). Les trois graphiques suivants montrent que, quelle que soit la

\footnotetext{
${ }^{6}$ Perrault (Guillaume), «Élu ou fonctionnaire : pourquoi l’UMP veut obliger à choisir », Le Figaro, 15 novembre 2006. Cette polémique resurgit régulièrement, la dernière en date ayant été lancée par Bruno Le Maire en septembre 2012 (sa critique s'adressait alors plus aux parlementaires qu'aux élus locaux, même s’il dénonçait de manière plus générale « la consanguinité entre politique et fonction publique $»)$.

${ }^{7}$ La distinction public/privé n'étant pas disponible dans les données du RNE pour les enseignants, ceux du privé ont été intégrés dans les chiffres produits pour prévenir toute critique sur ce point (le chiffre réel est par conséquent encore inférieur).
} 
taille de la commune, les maires "dominent» socialement leurs adjoints et tous les autres conseillers municipaux : quand les ouvriers - et les employés dans une moindre mesure - stagnent à des taux extrêmement faibles, la scène politique locale est dominée par le pôle supérieur de l'espace social, et ce d'autant plus que la taille de la commune et, corrélativement, le prestige de la fonction augmentent. Et si la différence entre « simple CM » et « adjoint » semble moins forte qu'entre «adjoint » et « maire », c'est tout simplement parce que, dans la catégorie « simple $\mathrm{CM}$ » se trouve noyée l'opposition, c'est-à-dire les quelques « rescapés » du système électoral qui donne une prime de $50 \%$ des sièges au vainqueur avant d'appliquer la proportionnelle, et qui sont en réalité celles et ceux qui auraient été aux premiers rangs de l'exécutif municipal si leur liste l'avait emporté.

Graphiques 2, 3 et 4 : Proportion de maires, adjoints et « simples » conseillers municipaux (CM) issus de trois catégories socioprofessionnelles, selon la taille de la commune

\section{Cadres et prof. intellect. sup}

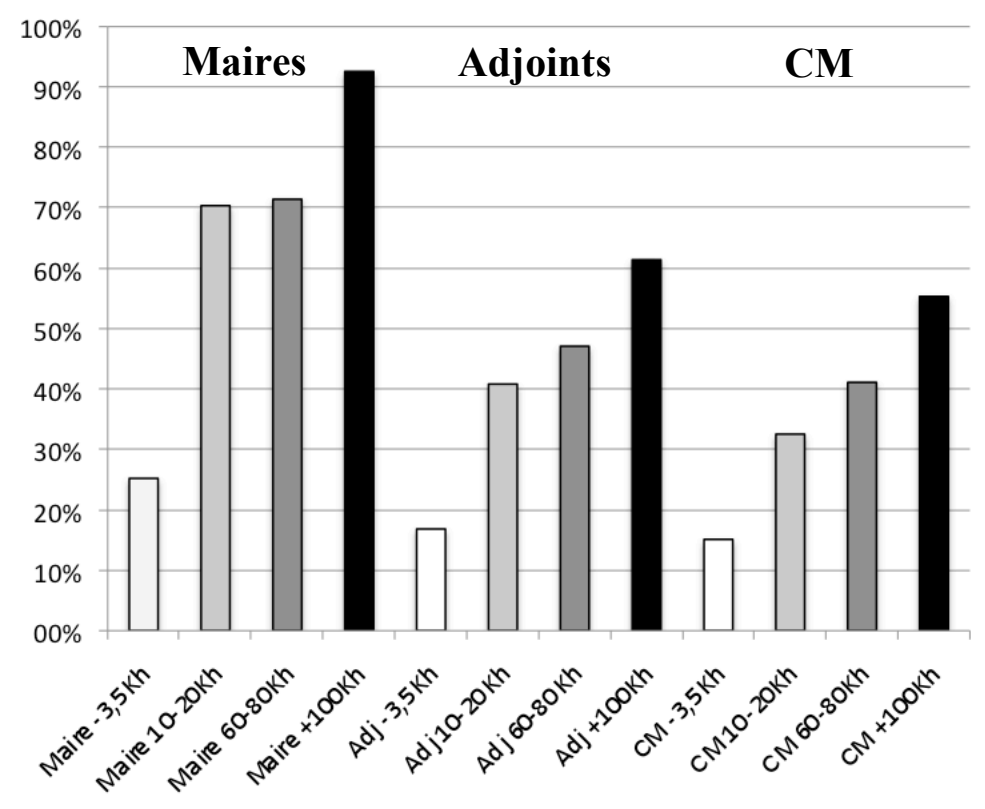

\section{Employés}

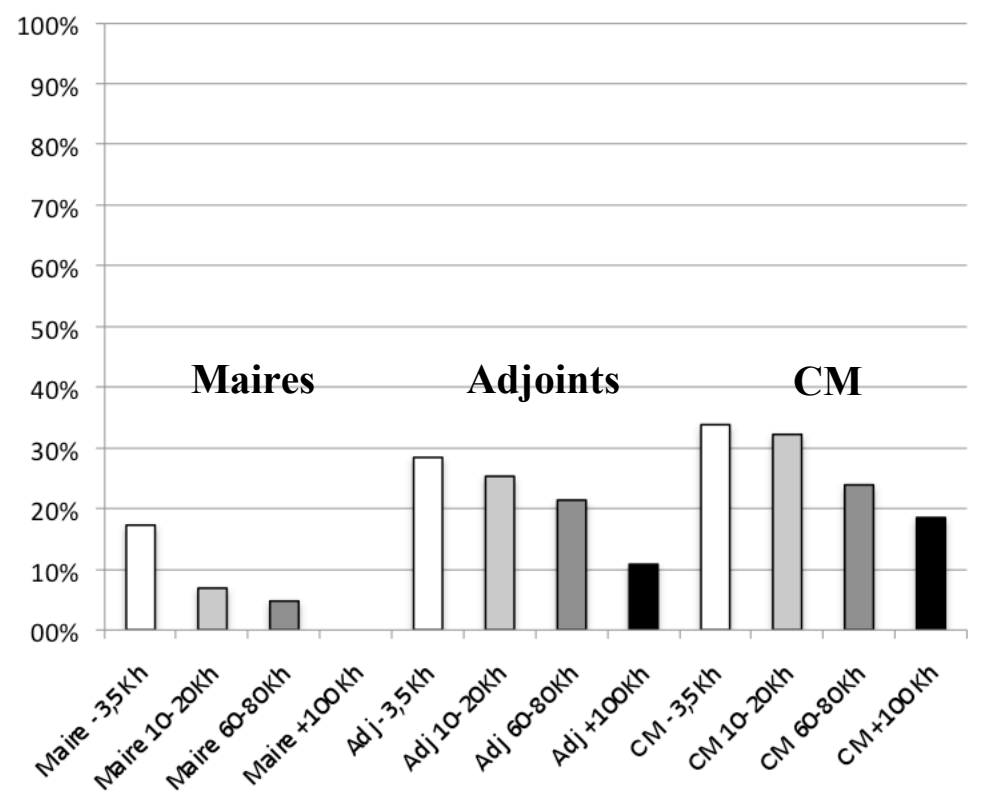




\section{Ouvriers}

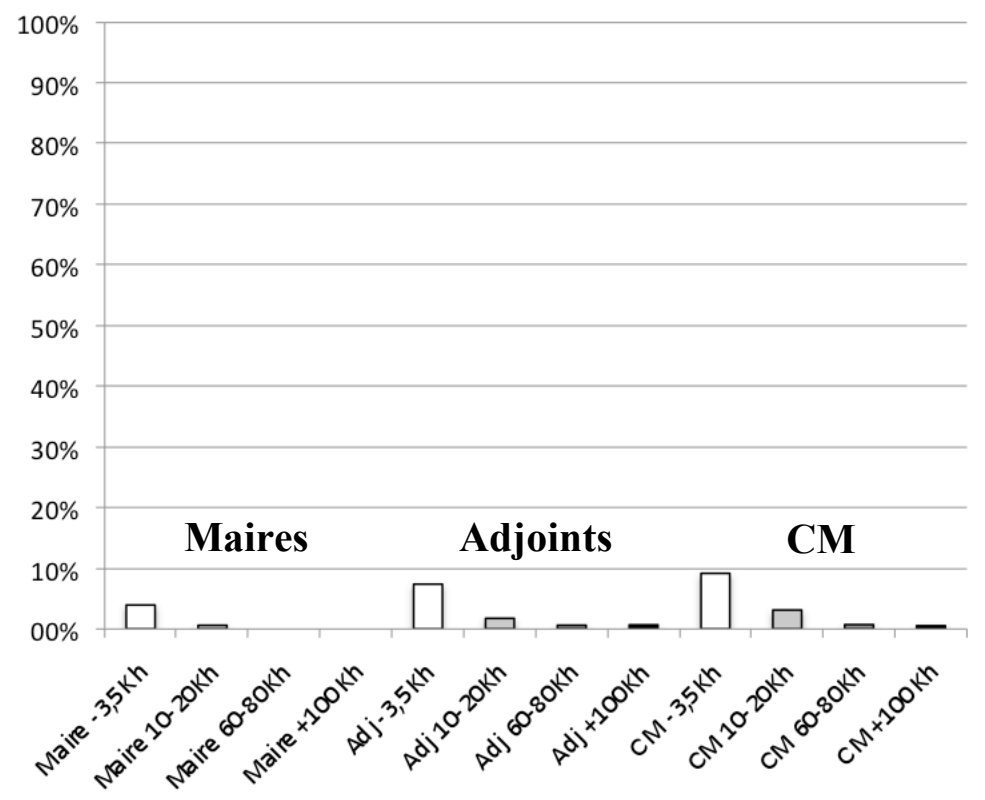

Ce type de graphique peut nous renseigner sur une autre caractéristique particulièrement intéressante : parmi les différentes catégories socioprofessionnelles d'actifs, seuls les cadres et professions intellectuelles supérieures (CPIS) et les agriculteurs sont d'autant plus représentés que l'on monte dans la hiérarchie des postes internes au conseil municipal (une proportion plus grande de maires que d'adjoints et de CM). Ces deux catégories partagent ce profil ascendant, qui correspond à une tendance à vouloir occuper les postes les plus prestigieux (même si, comme déjà évoqué plus haut, les profils selon la taille de la commune sont diamétralement opposés : les agriculteurs particulièrement représentés dans les petites communes, alors que les CPIS d'autant mieux représentés que la taille augmente). Or il se trouve que la catégorie des retraités est la seule qui ressemble aux deux décrites précédemment, ce qui nous permet - certes prudemment - de déduire que les retraités (dont le RNE ne donne aucun détail) sont plutôt d'anciens agriculteurs dans les petites communes et d'anciens cadres supérieurs quand la taille de la commune augmente, ce qui aurait tendance à renforcer encore le phénomène de confiscation des postes de pouvoir par les élites sociales locales.

L'analyse de l'appartenance politique des maires (comparés à leurs adjoints et aux autres conseillers municipaux) va dans le même sens : l'appartenance est d'autant plus marquée et affichée que l'on passe du simple conseiller au maire, et du village à la grande ville ${ }^{8}$. Une étude réalisée en 2013 sur un échantillon de 79 villes moyennes françaises ${ }^{9}$ a montré que les deux principaux partis politiques (PS et UMP) rassemblent $72,1 \%$ des maires de cet échantillon, $45,9 \%$ des 1 ers et $2^{\mathrm{e}}$ adjoints, $40,3 \%$ des adjoints suivants, et 33,6\% des autres conseillers municipaux. Les trois catégories «DVG», "DVD » et "Autres » sont d'autant moins utilisées que la taille et le prestige de la fonction augmentent : elles rassemblent seulement 16,4\% des maires (et seulement 6,90\% des maires de 60 à 80000 habitants) mais $48 \%$ des simples conseillers municipaux (36,5\% pour les communes de 60 à 80000 habitants).

\section{Quelques variations régionales sans logique évidente}

Les maires n'ont pas le même profil selon la région où ils exercent leur mandat. Le poids des différentes catégories socioprofessionnelles dans les régions est le produit complexe de la combinaison entre de nombreuses données, dont au moins le nombre de communes dans la région, leur répartition par taille, le nombre de maires par catégorie et la structure socioprofessionnelle de la population spécifique à cette

\footnotetext{
${ }^{8}$ C'est-à-dire le choix, au moment de la déclaration de candidature, d'un parti politique précis plutôt que d'un plus vague « divers droite » (DVD) ou « divers gauche » (DVG), ou encore de la case « autres ».

${ }^{9}$ Les résultats de cette enquête ont été présentés au congrès de l'AFSP en juillet 2013 : Koebel (Michel), « Logiques sociales dans la répartition des postes d'adjoint-e-s aux maires des villes moyennes françaises » et feront l'objet d'un article à paraître sur metropolitiques.eu en décembre 2013.
} 
région. Pour tenter de comparer ces données, nous avons choisi de retenir, pour quelques régions typiques de ce point de vue, le poids des maires de chaque catégorie par rapport à la population correspondante (nombre de maires d'une CSP pour 100000 habitants de cette CSP) et le rapport à la moyenne régionale de ce poids (cf. Tableau 3). Malgré ces précautions, on observe de fortes disparités régionales, et pas forcément celles que l'on attendait : lorsque l'on fait partie des catégories élevées dans la hiérarchie sociale, il vaut mieux habiter la Corse ou un territoire d'outre-mer pour optimiser ses chances de diriger une commune, et surtout pas l'île de France où la concurrence semble bien plus rude. C'est exactement l'inverse lorsque l'on est agriculteur. Lorsque l'on fait partie des professions intermédiaires, on a presque trois fois plus de chance d'être maire en Alsace que lorsque l'on brigue ce mandat dans la région Centre. Les ouvriers, même si leur représentation au sommet de la hiérarchie municipale est infime, sont bien mieux lotis en Île de France et dans la région Centre qu'en Alsace, en Corse ou dans le Limousin. Ni l'ancrage politique, ni le taux de féminisation des maires, très variables selon les régions, ni aucune des autres données disponibles ne permettent de découvrir une logique simple entre ces différents poids et indices. De même, lorsque l'on compare les communes rurales et urbaines, en dehors de la quasi disparition de certaines catégories (agriculteurs et ouvriers), les écarts sont très variables d'une région à l'autre sans aucune proportionnalité évidente. L'une des explications - certes pas tout à fait satisfaisante - pourrait être liée à l'héritage politicoculturel des régions et des communes qui les composent. En effet, il existe des effets puissants d'inertie dans le domaine politique, qu'illustre déjà la simple statistique concernant le renouvellement du personnel politique local : en 2008, 59,7\% des maires sortants ont été réélus en France... Ce fort pourcentage peut nous interroger également sur la réalité de l'appartenance sociale des maires qui font carrière en politique : peut-on encore considérer qu'ils sont « ouvriers » ou « employés » quand ils enchaînent plusieurs mandats et qu'ils en cumulent d'autres, pour devenir de véritables professionnels de la politique ? L'INSEE ne s'y est pas trompée : elle classe les permanents politiques dans la catégorie des cadres et professions intellectuelles supérieures. Certes les compétences préalables des candidats sont des ressources importantes pour se faire élire ; mais l'exercice d'un mandat (et a fortiori de plusieurs) développe aussi au fil des années les compétences de celui qui l'exerce, et lui apporte un capital social et symbolique bien supérieur et très éloigné de celui dont bénéficie un ouvrier s'il l'était resté (et sauf s'il s'investit fortement dans le monde syndical). Il en va de même pour les ressources financières de ces élus, qui dépassent largement le salaire qu'ils obtenaient en exerçant leur précédent métier.

Tableau 3 : Nombre de maires pour 100000 habitants selon la catégorie socioprofessionnelle pour quelques régions françaises (en italiques : poids de cette représentation par rapport à la moyenne ${ }^{10}$ )

\begin{tabular}{|l|c|c|c|c|c|c|c|}
\hline CSP (8 postes) & Alsace & Centre & Corse & $\begin{array}{c}\text { Ile-de- } \\
\text { France }\end{array}$ & Limousin & PACA & Outre-Mer \\
\hline \multirow{2}{*}{ Agriculteurs exploitants } & 930,7 & 1386,1 & 1007,7 & 1182,5 & 789,7 & 456,3 & 30,6 \\
& 15,64 & 15,89 & 7,35 & 87,58 & 6,67 & 19,36 & 2,86 \\
\hline Artisans, commerçants, chefs & 93,2 & 144,3 & 138,6 & 27,3 & 155,8 & 53,2 & 11,3 \\
d'entreprise & 1,57 & 1,65 & 1,01 & 2,02 & 1,32 & 2,26 & 1,05 \\
\hline Cadres et professions & 195,3 & 216,2 & 672,9 & 23,2 & 359,5 & 71,8 & 83,9 \\
intellectuelles supérieures & 3,28 & 2,48 & 4,90 & 1,72 & 3,04 & 3,05 & 7,83 \\
\hline \multirow{2}{*}{ Professions intermédiaires } & 89,4 & 48,2 & 81,9 & 10,7 & 90,1 & 15,9 & 14,8 \\
& 1,50 & 0,55 & 0,60 & 0,79 & 0,76 & 0,67 & 1,38 \\
\hline \multirow{2}{*}{ Employés } & 48,6 & 41,2 & 80,8 & 5,2 & 82,2 & 10,6 & 8,2 \\
& 0,82 & 0,47 & 0,59 & 0,39 & 0,69 & 0,45 & 0,76 \\
\hline \multirow{2}{*}{ Ouvriers } & 6,2 & 22,4 & 7 & 3,2 & 6,2 & 2,1 & 0,00 \\
& 0,10 & 0,26 & 0,05 & 0,24 & 0,05 & 0,09 & 0,00 \\
\hline \multirow{2}{*}{ Retraités } & 57,1 & 92,5 & 188,7 & 19,5 & 126,8 & 28,8 & 12,9 \\
& 0,96 & 1,06 & 1,38 & 1,44 & 1,07 & 1,22 & 1,21 \\
\hline Autres professions ou sans & 5,7 & 57,4 & 44,1 & 6,1 & 54,2 & 6,9 & 2,9 \\
profession déclarée ou étudiants & 0,10 & 0,66 & 0,32 & 0,45 & 0,46 & 0,29 & 0,27 \\
\hline Moyenne & $\mathbf{5 9 , 5}$ & $\mathbf{8 7 , 2}$ & $\mathbf{1 3 7 , 2}$ & $\mathbf{1 3 , 5}$ & $\mathbf{1 1 8 , 4}$ & $\mathbf{2 3 , 6}$ & $\mathbf{1 0 , 7}$ \\
\hline
\end{tabular}

\footnotetext{
${ }^{10}$ Lecture : en Alsace, on compte 930,7 maires agriculteurs pour 100000 agriculteurs, ce qui représente presque 15,64 fois la moyenne (59,5 maires pour 100000 habitants).
} 


\section{Peu d'évolution sur l'âge et le sexe des maires, malgré l'injonction à la parité}

Concernant l'âge et le sexe, rien de bien nouveau. Après les élections de 2001, Catherine Achin constatait, statistiques à l'appui (elle n'étudiait que les conseils municipaux des villes de plus de 3500 habitants), que " l'entrée massive des femmes en politique à l'échelon municipal a pu conduire à un élargissement de la base sociale de recrutement du personnel politique ${ }^{11} \gg$. Certes la proportion de femmes maires a légèrement augmenté en 2001 puis en 2008, mais, comme la loi sur la parité ne peut s'appliquer que là où existe une liste et que le poste de maire est unique, la domination masculine joue encore à plein, puisque là où la parité est censée s'appliquer - c'est-à-dire dans les communes de plus de 3500 habitants -, les femmes ne représentent qu'à peine plus de 10\% des maires. Quelques variations existent selon la taille et la catégorie socioprofessionnelle. Par exemple, en milieu rural, plus le village est petit, plus la fonction de maire est féminisée (jusqu'à atteindre plus de $20 \%$ dans les villages de moins de 50 habitants) ; en milieu urbain, on observe aussi une féminisation un peu plus élevée au-dessus de 50000 habitants, mais elle ne concerne qu'un peu plus de 100 communes en France... On retrouve classiquement plus de femmes maires chez les employés et sans profession que chez les ouvriers (respectivement 29,7,35,1 et 7,1\%); les catégories les moins féminisées après les ouvriers sont les artisans, commerçants et chefs d'entreprises $(7,2 \%)$ et les agriculteurs $(8,0 \%)$, les autres catégories se situant autour de $12 \%$. Mais le constat global reste sans appel : la place laissée aux femmes dans la direction des équipes municipales reste minime. On aurait pu espérer un rééquilibrage au profit des femmes au sein des exécutifs municipaux, ceux-ci étant soumis aussi depuis les dernières élections à une exigence de parité. Mais là aussi, le constat est alarmant : la parité reste d'abord mathématique et la domination masculine prend la voie du domaine de compétences pour s'exercer, en suivant des logiques que l'on n'imaginait pas être encore aussi vivaces dans le domaine politique : dans un échantillon de 890 adjoints de villes moyennes françaises, on constate que l'enfance/la petite enfance/la famille, l'animation/la vie associative, le social et les affaires scolaires reviennent très majoritairement aux femmes (respectivement à 93, 78, 77 et 70\%), alors que les finances et l'urbanisme (domaines les plus prestigieux, à en juger par le rang des adjoints correspondants), les travaux et le sport reviennent aux hommes (respectivement à 78, 75, 69 et 69\%). Cette répartition - presque caricaturale tant elle reflète les préjugés classiques - s'accentue encore quand la taille de la commune et donc le prestige de la fonction augmentent ${ }^{12}$ (et cette discrimination se perpétue avec la complicité - le plus souvent bien involontaire des femmes elles-mêmes).

\section{Graphique 5 : Âge des maires selon le sexe (en pointillés : actifs seulement)}

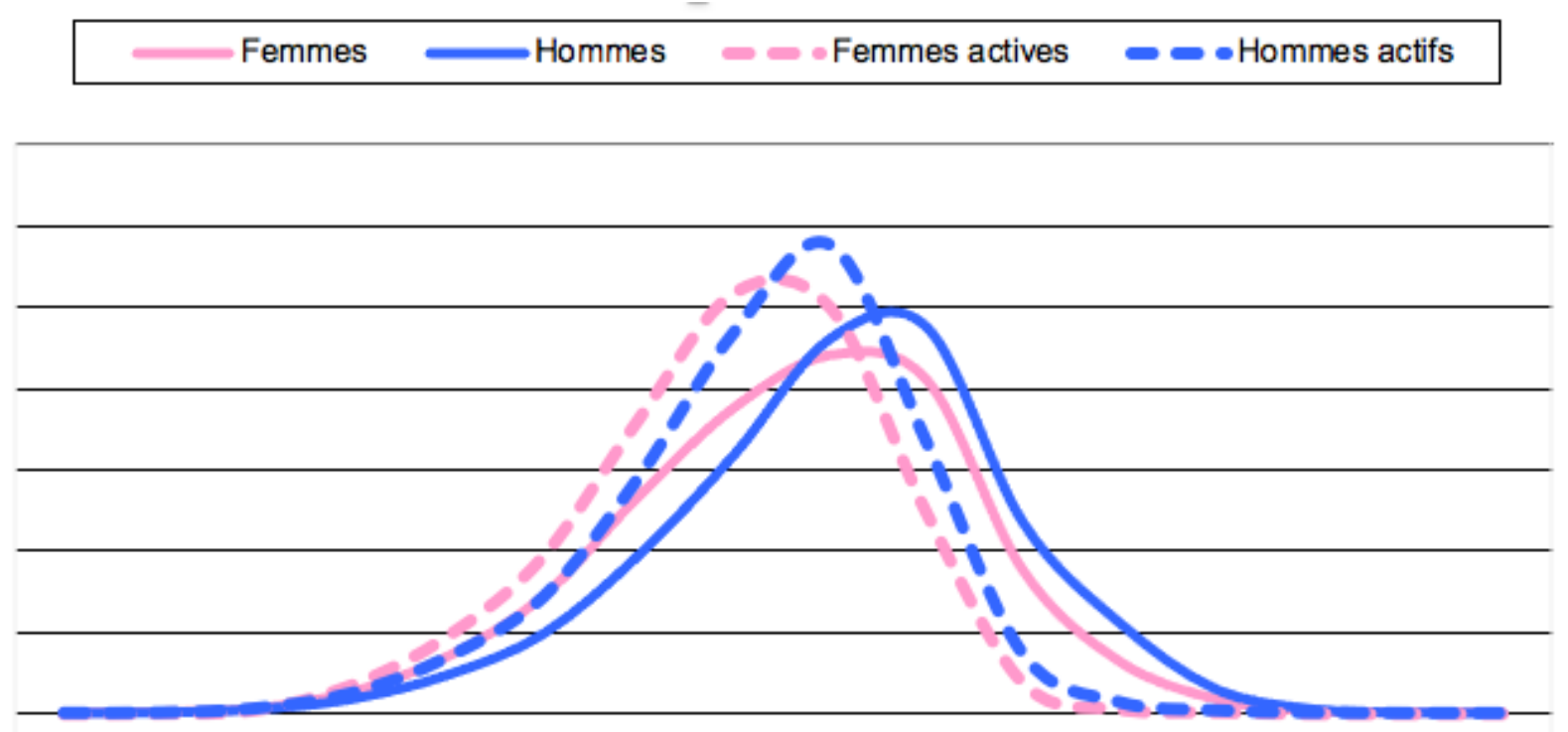

\footnotetext{
${ }^{11}$ Achin (Catherine) : 2001. « Démocratisation du personnel politique et parité : un premier bilan », Mouvements, 2001/5 $\mathrm{n}^{\circ} 18$, p. 57-61. DOI : 10.3917/mouv.018.0057

${ }^{12}$ Koebel (Michel), « Les hiérarchies du pouvoir local », Savoir/Agir, 25, septembre 2013 (p. 31-37).
} 
Constat à peu près identique en ce qui concerne l'âge : les « jeunes » restent exclus des responsabilités locales, et on ne compte que 3,7\% de moins de 40 ans chez les maires. Certes un âge avancé (l'une des rares raisons, avec la maladie et la mort, qui peut faire abandonner la politique, comme le disait Gaxie) fait très vite chuter les pourcentages, mais l'âge d'or de l'exercice du pouvoir local se situe tout de même entre 55 et 70 ans (et ce, en début de mandat, puisqu'en fin de mandat, il faut évidemment ajouter six ans) (cf. Graphique 5). L'âge moyen des femmes maires n'est inférieur à leurs homologues masculins que d'un peu plus de deux ans $(55,1$ contre 57,5), et la forte augmentation de la part des retraités parmi les maires, conjuguée au faible renouvellement des maires d'une élection à l'autre, ne vont pas dans le sens d'un rajeunissement.

\section{Conclusion}

Au terme de cette analyse, s'il fallait résumer le profil social des maires de France, il serait assez difficile d'échapper à certains des clichés qui sont souvent propagés par ceux qui n'ont jamais pris le temps d'analyser les données de manière approfondie, des données pourtant patiemment accumulées par les préfectures et remontées au niveau national sans que personne (ou presque) ne s'y intéresse. Si l'on admet - du fait notamment des règles de scrutin et du traitement différencié des communes en fonction de leur taille - que le pouvoir local est fortement concentré sur les maires et qu'il est proportionnel à la taille des communes, alors on peut affirmer que l'essentiel de ce pouvoir en France reste accaparé par le pôle le plus élevé de la hiérarchie sociale et professionnelle, et dont les représentants sont quasi exclusivement des hommes plutôt âgés et encartés. Les évolutions récentes, liées à la loi sur la parité ou à la démographie, ne font pas fondamentalement varier ce profil, même si l'on peut constater certaines variations régionales.

\section{Résumé}

L'analyse des données du Répertoire national des élus (élections municipales de 2008) et leur mise en perspective avec d'autres données, démographiques et issues de plusieurs enquêtes récentes, permettent de développer divers aspects du profil social des maires de France et de son évolution dans le temps, et de faire apparaître les logiques sociales qui le font plus stagner que se transformer. Les postes les plus prestigieux restent aux mains d'une élite sociale et politique locale, masculine et âgée, à peine transformée par les évolutions démographiques et les injonctions à la parité. 